\title{
Incremental Pattern-Based Coinduction for Process Algebra and Its Isabelle Formalization
}

\author{
Andrei Popescu and Elsa L. Gunter \\ University of Illinois at Urbana-Champaign
}

\begin{abstract}
We present a coinductive proof system for bisimilarity in transition systems specifiable in the de Simone SOS format. Our coinduction is incremental, in that it allows building incrementally an a priori unknown bisimulation, and pattern-based, in that it works on equalities of process patterns (i.e., universally quantified equations of process terms containing process variables), thus taking advantage of equational reasoning in a "circular" manner, inside coinductive proof loops. The proof system has been formalized and proved sound in Isabelle/HOL.
\end{abstract}

\section{Introduction}

Bisimilarity is arguably the most natural equivalence on interactive processes. Assuming process transitions are labeled by (observable) actions $a$, processes $P$ and $P^{\prime}$ are bisimilar iff: (I) whenever $P$ can $a$-transit to a process $Q, P^{\prime}$ can also $a$-transit to some process $Q^{\prime}$ such that $P^{\prime}$ and $Q^{\prime}$ are again bisimilar; (II) and vice versa; (III) and so on, indefinitely (as in an infinite game).

The above informal description of the bisimilarity relation can of course be made rigorous by defining bisimilarity to be the largest bisimulation, i.e., the largest relation $\theta$ for which (I) and (II) hold (with "bisimilar" replaced by "in $\theta$ "). But the largest-fixpoint description loses (at least superficially) the gametheoretic flavor of the intuitive description, so we stick to the latter for a while. How would one go about proving that $P$ and $Q$ are bisimilar? Well, if one were allowed an infinite proof, one could try to show that each transition of $P$ is matched by a transition of $Q$ so that the continuations $P^{\prime}$ and $Q^{\prime}$ are (claimed to be) bisimilar (and vice versa), and then prove the bisimilarity claims about all pairs of continuations $P^{\prime}$ and $Q^{\prime}$, and so on. This way, one would build an infinite tree whose nodes contain bisimilarity claims about pairs of processes. Now assume that, while expanding the tree, one encounters a repetition of a previous claim (that appeared on an ancestor node). A reasonable "optimization" of the infinite proof would then be to stop and "seal" that node, because the bisimilarity argument for its ancestor can be repeated ad litteram. In other words, one may take the (yet unresolved!) goal of the ancestor as a hypothesis, which discharges the repetitive goal - this is the upside of trying to build an infinite proof: non-well-foundedness (i.e., circularity) works in our advantage. Assume now one finds such repetitions on all paths when building the tree. Then our bisimilarity proof is done! In terms of the fixpoint definition, we have

L. Ong (Ed.): FOSSACS 2010, LNCS 6014, pp. 109 127, 2010.

(C) Springer-Verlag Berlin Heidelberg 2010 
proved that the pair $(P, Q)$ of processes located at the root are bisimilar by coinduction, i.e., by exhibiting a bisimulation that contains $(P, Q)$. In terms of proof engineering however, the needed bisimulation did not appear out of nowhere, but was built incrementally from the goal, essentially by an exploration that discovered a regular pattern for an infinite proof tree. In fact, coinductive proofs are intuitively all about discovering regular patterns.

This paper provides formal support for this intuition. Here is an illustration of our approach, for a mini process calculus. Fix a set of actions act with a given silent action $\tau \in$ act and a map on act $\backslash\{\tau\}, a \mapsto \bar{a}$, such that $\overline{\bar{a}}=a$ for all $a \in A$ ct. The processes $P$ are generated by the grammar: $P::=0|a . P| P|Q| ! P$. Thus, we have idle process, action prefix, parallel composition, and replication. "!" binds more strongly than "|". The behavior of processes is specified by the following labeled transition system:

$$
\begin{gathered}
\frac{\cdot}{a . P \stackrel{a}{\rightsquigarrow} P} \text { (PREF) } \frac{P_{0} \stackrel{a}{\rightsquigarrow} Q_{0}}{P_{0}\left|P_{1} \stackrel{a}{\rightsquigarrow} Q_{0}\right| P_{1}} \text { (PARL) } \frac{P_{1} \stackrel{a}{\rightsquigarrow} Q_{1}}{P_{0}\left|P_{1} \stackrel{a}{\rightsquigarrow} P_{0}\right| Q_{1}} \text { (PARR) } \\
\frac{P_{0} \stackrel{a}{\rightsquigarrow} Q_{0} \quad P_{1} \stackrel{a}{\rightsquigarrow} Q_{1}}{P_{0}\left|P_{1} \stackrel{\tau}{\rightsquigarrow} Q_{0}\right| Q_{1}} \text { (PARS) } \frac{P^{\stackrel{a}{\rightsquigarrow} Q}}{! P \stackrel{a}{\rightsquigarrow} ! P \mid Q} \text { (REPL) } \frac{P \stackrel{a}{\rightsquigarrow} Q_{0} \quad P \stackrel{a}{\rightsquigarrow} Q_{1}}{! P \stackrel{\tau}{\rightsquigarrow} ! P \mid\left(Q_{0} \mid Q_{1}\right)} \text { (REPLS) }
\end{gathered}
$$

We may wish to prove in this context that parallel composition is associative and commutative and that replication absorbs self-parallel composition, i.e., that $\left(P_{0} \mid P_{1}\right)\left|P_{2}=P_{0}\right|\left(P_{1} \mid P_{2}\right), P_{0}\left|P_{1}=P_{1}\right| P_{0}$, and $P \mid ! P=! P$ for all processes $P_{0}, P_{1}, P_{2}, P$, where we write "=" for strong bisimilarity. In fact, assume we already proved the first two facts and are left with proving the third, $P \mid ! P=! P$. For this, we first check to see if the equations we already know so far (associativity and commutativity of |) imply this new one by pure equational reasoning - no, they don't. This means we cannot discharge the goal right away, and therefore we need to perform unfoldings of the two terms in the goal. We unfold $P \mid ! P$ and $! P$ until we reach hypotheses involving only process meta-variables. The upper side of Figure 1 contains all possible derived rules (i.e., compositions of primitive rules in the system, all the way down to non-decomposable hypotheses) that can be matched by $P \mid ! P$ in order to infer a transition from $P \mid ! P$. And, similarly, the lower side for the term $! P$ - in this latter case, the matched derived

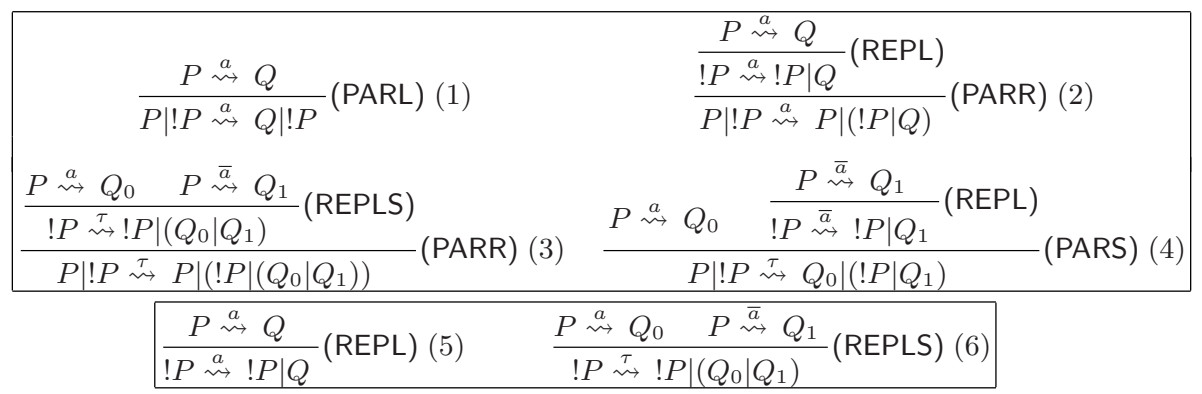

Fig. 1. The matching derived rules for $P \mid ! P$ and $! P$ 
rules coincide with the matched primitive rules. To see how the derived rules are obtained, the figure shows whole derivation trees, but we only care about the leaves and the roots of these trees.

Next, we try to pair these derived rules (upper versus lower), by the accordance of their hypotheses and their transition labels. The only valid pairing possibilities are: (1) with (5), (2) with (5), (3) with (6), and (4) with (6). The targets of the conclusions of the rules in these pairs yield four new goals: (i) $Q|! P=! P| Q$; (ii) $P|(! P \mid Q)=! P| Q$; (iii) $P\left|\left(! P \mid\left(Q_{0} \mid Q_{1}\right)\right)=! P\right|\left(Q_{0} \mid Q_{1}\right)$; (iv) $Q_{0}\left|\left(! P \mid Q_{1}\right)=! P\right|\left(Q_{0} \mid Q_{1}\right)$. The original goal, $P \mid ! P=! P$, is replaced by the above four goals, and is also henceforth taken as a hypothesis. Notice that our goals are generic, i.e., universally quantified over the occurring process meta-variables, $P, Q, Q_{0}, Q_{1}$. Now, equational reasoning (by standard equational rules, including substitution) with hypothesis $P \mid ! P=! P$ together with the already known lemmas $\left(P_{0} \mid P_{1}\right)\left|P_{2}=P_{0}\right|\left(P_{1} \mid P_{2}\right)$ and $P_{0}\left|P_{1}=P_{1}\right| P_{0}$ is easily seen to discharge each of the remaining four goals, and the proof is done.

Why is this proof valid, i.e., why does it represent a proof of the fact that, for all process terms $P, P \mid ! P$ and $! P$ are bisimilar? The rigorous justification for this is the topic of this paper. But the short answer has to do with our previous discussion on discovering patterns: the above is really a proof by coinduction (on universally quantified equalities of terms up to equational closure), which builds incrementally the relation representing the coinductive argument. Notice the appearance of circular reasoning: a goal that cannot be locally discharged is expanded according to the SOS definition transition relation and becomes a hypothesis. In this particular example, the proof is finished after only one expansion, but the process of expanding the goals with taking them as hypotheses may in principle continue, obtaining arbitrarily large proof trees.

We show that deductions such as the above are sound for a wide class of process algebras - those specifiable by SOS rules in the de Simone format [1]. Our results have been given a formalization in Isabelle/HOL [3], which was desirable for two reasons: first, the very technical constructions (especially in Sec. 4) and arguments (in both Secs. 3 and 4) were asking for a means to be absolutely sure of their correctness; second, the formalization has the potential of leading to an implementation of a coinductive tool. Here is the structure of this paper. The rest of this section establishes some notation. Sec. 2 discusses our representation of the de Simone format. Secs. 3 and 4 contain our original theoretic contribution: incremental proof systems for bisimilarity - Sec. 3 for standard bisimilarity, Sec. 4 for universally quantified bisimilarity equations. Sec. 5 discusses related and future work. More details on our Isabelle scripts and on various other technical topics, as well as more examples, can be found in the technical report [28, to which we occasionally refer from this paper. 28] is an identical copy of this paper, except that it has an appendix with more details. The Isabelle scripts can be found at http://hdl.handle.net/2142/14857 in both html-browsable and pdf formats (App. C in 28, has details about the scripts.) 
Conventions and notations. By "Isabelle", here we mean "Isabelle/HOL". We present our work in the usual mathematical language, but partly employ the Isabelle dialect of this language in order to allow the interested reader to easily relate this paper with our Isabelle formal proofs. (We believe that this choice does not decrease readability, since the Isabelle notation is very close to standard mathematical notation and also occasionally allows for clear and concise formulations, as, e.g., with datatypes and records. A priori familiarity with the Isabelle dialect is not required from the reader.)

Isabelle distinguishes between a type and a set, but the set-theoretical-oriented reader is free to ignore this distinction; as a matter of syntax though, membership to a type is denoted by "::" and membership to a set by " $\in$ ". nat is the type of naturals. Given types $\alpha$ and $\beta, \alpha \times \beta$ is their product type, $\alpha \Rightarrow \beta$ the type of functions between $\alpha$ and $\beta, \alpha \underline{\text { list }}$ the type of lists of items in $\alpha$, and $\alpha$ set the type of sets of items in $\alpha 1$ fst and snd are the two projections from $\alpha \times \beta$. [] is the empty list and $\left[a_{0}, \ldots, a_{n-1}\right]$ the list consisting of the $n$ indicated items; given a list $L$ and $i<$ length $L, L ! i$ is the $(i+1)$-th element in $L$ (thus, the first element is $L ! 0)$. The operator set : $\alpha \underline{\text { list }} \Rightarrow \alpha \underline{\text { set }}$ gives the set of the items appearing in a list. A list $L$ is said to be nonrepetitive if $L ! i \neq L ! j$ for all $i, j<$ length $L$ with $i \neq j$. For readability, we consistently use: sans serif fonts for constants, such as length and set; boldface for types, such as nat; underlined boldface for type constructors, such as list and $\underline{\text { set. }}$.

\section{Syntax and Operational Semantics of Processes}

Process variables, terms and substitution. We fix the following types: param, of parameters, ranged over by $p$; opsym, of operation symbols (opsyms for short), ranged over by $f, g$; var, of (process) variables, ranged over by $X, Y, Z$ - this latter type is assumed to be infinite. The type term, of (process) terms, ranged over by $P, Q, R, T, S, U, V$, is defined as an Isabelle datatype (i.e., initial algebra): DATATYPE term = Var var $\mid$ Op opsym (param list) $($ term list).

Thus, a term can have any opsym at the top, applied to any list of parameters and any list of terms (of any length), without being subject to further well-formedness conditions. Hence an opsym $f$ does not have an a priori associated numeric rank $(m, n)$ (indicating that $f$ takes $m$ parameters and $n$ terms). Rather, we allow in term the whole pool of all possible terms under all possible rankings of the operation symbols. This looseness w.r.t. terms is admittedly a formalization shortcut (fitting nicely the Isabelle simply-typed framework), but is completely unproblematic for the concepts and results of this paper: while an SOS specification of a transition system will of course employ only certain (possibly overloaded) ranks for the opsyms, the unused ranks will be harmless, since they will not affect transitions or bisimilarity.

\footnotetext{
${ }^{1}$ Note the use of postfix notation for type constructors - this is not standard mathematically, but is intuitive, as it matches natural language: while an element of int is an integer, an element of int list is an integer list.
} 
$\sigma$ and $\tau$ will range over var $\Rightarrow$ term. We consider the operators:

- vars :: term $\Rightarrow$ var $\underline{\text { set}}$, giving the set of variables occurring in a term.

- _[_] :: term $\times(\operatorname{var} \Rightarrow$ term $) \Rightarrow$ term, such that $T[\sigma]$ is the term obtained from $T$ by substituting all its variables $X$ by $\sigma X$.

Next we represent the meta-SOS notion of a transition-system specification [15 26]. Given any type $\alpha$, the type $\alpha \underline{\text { ftrans, }}$, of formal $\alpha$-transitions, consists of pairs, written $k \rightsquigarrow l$, with $k, l:: \alpha$, where $k$ is called the source and $l$ the target. We fix a type act, of actions, ranged over by $a, b$.

Rules syntax. The type rule, of (SOS-)rules, ranged over by $r l$, is defined to be the following record type (i.e., a product with named projections): RECORD rule=

$$
\begin{aligned}
& \text { hyps :: (var } \underline{\text { ftrans }}) \underline{\text { list }} \text { (read "hypotheses") } \\
& \text { cnc :: term } \underline{\text { trans }} \text { (read "conclusion") } \\
& \text { side :: (nat } \Rightarrow \text { act) } \Rightarrow \text { act } \Rightarrow \text { bool (read "side-condition") }
\end{aligned}
$$

The hypotheses and the conclusions of our rules are therefore formal transitions between variables, and between terms, respectively. I.e., for any rule $r l$ :

- hyps $r l$ has the form $\left[X X_{0} \rightsquigarrow Y_{0}, \ldots, X X_{n-1} \rightsquigarrow Y_{n-1}\right]$, with $X X_{j}, Y_{j}$ variables; - cnc $r l$ has the form $S \rightsquigarrow T$, with $S$ and $T$ terms.

One can visualize $r l$ as

$$
\frac{X X_{0} \rightsquigarrow Y_{0}, \ldots, X X_{n-1} \rightsquigarrow Y_{n-1}}{S \rightsquigarrow T}[\lambda a s, b \text {. side } r l \text { as } b]
$$

where as :: nat $\Rightarrow$ act and $b$ :: act. Actually, we think of $r l$ as follows:

$$
\left.\frac{X X_{0} \stackrel{a s 0}{\rightsquigarrow} Y_{0}, \ldots, X X_{n-1} \stackrel{\text { as }(n-1)}{\rightsquigarrow} Y_{n-1}}{S \stackrel{b}{\rightsquigarrow} T} \text { [side } r l \text { as } b\right]
$$

Note however that the side condition side $r l$ is (for now) allowed to take into consideration the whole function as, and not only its first $n$ values as $0, \ldots$, as $(n-$ 1 ), as one would expect - this is corrected below by "saneness".

Given a rule $r l$ with hyps $r l$ and cnc $r l$ as above, we write: theXXs $r l$, for the variable list $\left[X X_{0}, \ldots, X X_{n-1}\right]$; theYs $r l$, for the variable list $\left[Y_{0}, \ldots, Y_{n-1}\right]$; theS $r l$, for the term $S$; theT $r l$, for the term $T$.

A rule $r l$ is said to be sane if the following hold:

-(1) theYs $r l$ is nonrepetitive;

-(2) $\operatorname{set}($ theXXs $r l) \subseteq \operatorname{vars}($ theS $r l)$;

-(3) vars(theS $r l) \cap \operatorname{set}($ theYs $r l)=\emptyset$;

-(4) $\operatorname{vars}($ the $T r l) \subseteq \operatorname{vars}($ the $S r l) \cup \operatorname{set}($ theYs $r l)$;

-(5) $\forall a s, a s^{\prime} .(\forall i<$ length(theYs $r l)$. as $\left.i=a s^{\prime} i\right) \longrightarrow$ side $r l$ as = side $r l a s^{\prime}$.

A rule $r l$ is said to be amenable if theS $r l$ has the form Op $f p s\left[\operatorname{Var} X_{0}, \ldots, \operatorname{Var}\right.$ $\left.X_{m-1}\right]$, where $f$ is an opsym, $p s$ a list of parameters, and $\left[X_{0}, \ldots, X_{m-1}\right]$ a nonrepetitive list of variables. Given an amenable rule $r l$ as above, we write thef $r l$ for $f$, theps $r l$ for $p s$, and theXs $r l$ for $\left[X_{0}, \ldots, X_{m-1}\right]$.

Saneness expresses a natural property for well-behaved SOS rules: Think of a term $S$ as a generic composite process, built from its unspecified components 
(its variables) by means of opsyms. Then a sane rule is one that describes the behavior of the composite $S$ in terms of the behavior of (some of) its components: condition (2) says that indeed the hypotheses refer to the components, (1) and (3) that the hypotheses only assume that some components transit "somewhere" (without any further information), (4) that the resulted continuation of the composite depends only on the components and their continuations, and (5) that the side-condition may only depend on the action labels of the hypotheses and of the conclusion. In addition, amenability asks that the composite process $S$ be obtained by a primitive operation $f$ applied to unspecified components. The conjunction of saneness and amenability is precisely the de Simone format requirement 11, hence we call a rule de Simone if it is sane and amenable.

Running example. We show what the example in the introduction becomes under our representation. Assume that act is an unspecified type with constants - :: act $\Rightarrow$ act and $\tau::$ act such that $\overline{\bar{a}}=a$ for all $a \neq \tau$. Define the relation sync : : act $\Rightarrow$ act $\Rightarrow$ act $\Rightarrow$ bool by sync $a b c=(a \neq \tau \wedge b \neq \tau \wedge \bar{a}=b \wedge c=\tau)$. We take opsym to be a three-element datatype Pref $\mid$ Par | Repl and param to be act. For readability, in our running example (including throughout the future continuations of this example), for all $X::$ var, $S, T::$ term and $a::$ act, we use the following abbreviations: $X$ for $\operatorname{Var} X$; a.S for Op Pref $[a][S] ; S \mid T$ for Op Par [] [T,S]; ! $S$ for Op Repl [] [S].

$R l s$ consists of the rules $\left\{\mathrm{PREF}_{a} . a::\right.$ act $\} \cup\{\mathrm{PARL}, \mathrm{PARR}, \mathrm{PARS}, \mathrm{REPL}, \mathrm{REPLS}\}$ listed below, where $X, Y, X_{0}, X_{1}, Y_{0}, Y_{1}$ are fixed distinct variables.

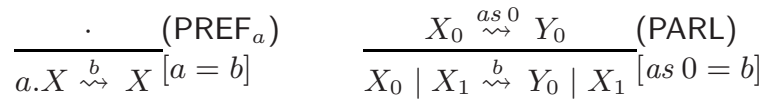

$$
\begin{aligned}
& \frac{X_{0} \stackrel{a s 0}{\rightsquigarrow} Y_{0}}{X_{1}\left|X_{0} \stackrel{b}{\rightsquigarrow} X_{1}\right| Y_{0}}[\text { (PARR) } 0=b] \quad \frac{X_{0} \stackrel{\text { as } 0}{\rightsquigarrow} Y_{0} \quad X_{1} \stackrel{a s 1}{\rightsquigarrow} Y_{1} \text { (PARS) }}{X_{0}\left|X_{1} \stackrel{b}{\rightsquigarrow} Y_{0}\right| Y_{1}} \text { [sync (as 0) (as 1) b] } \\
& \frac{X \stackrel{\text { as } 0}{\rightsquigarrow} Y \text { (REPL) }}{! X \stackrel{b}{\rightsquigarrow} ! X \mid Y}[\text { as } 0=b] \quad \frac{X \stackrel{\text { as } 0}{\rightsquigarrow} Y_{0} \quad X \stackrel{\text { as } 1}{\rightsquigarrow} Y_{1}}{! X \stackrel{b}{\rightsquigarrow} ! X \mid\left(Y_{0} \mid Y_{1}\right)}[\text { RePLS }(\text { as } 0)(\text { as } 1) b]
\end{aligned}
$$

For listing the rules, we employed the previously discussed visual representation. E.g., the formal description of PARS is ( hyps $=\left[X_{0} \rightsquigarrow Y_{0}, X_{1} \rightsquigarrow Y_{1}\right]$; cnc $=$ $\left(X_{0}\left|X_{1} \rightsquigarrow Y_{0}\right| Y_{1}\right)$; side $=(\lambda a s, b$. sync $($ as 0$)($ as 1$\left.) b) \mid\right)$. All the rules in this example are easily seen to be de Simone.

Rules semantics. We fix Rls, a set of de Simone rules. The one-step transition relation induced by $R l s$ on terms is a (curried) ternary relation step :: term $\Rightarrow$ act $\Rightarrow$ term $\Rightarrow$ bool, where we write $P \stackrel{a}{\rightsquigarrow} Q$ instead of step $P$ a $Q$, defined inductively by the following clause:

- if $r l \in R l s, \sigma(($ theXXs $r l) ! j) \stackrel{a s j}{\sim} \sigma(($ theYs $r l) ! j)$ for all $j<$ length(theYs $r l)$, and side $r l$ as $b$ holds, then (theS $r l)[\sigma] \stackrel{b}{\rightsquigarrow}$ (theT $r l$ ) $[\sigma]$ (where $\sigma::$ var $\Rightarrow$ term, as :: nat $\Rightarrow$ act, and $b::$ act).

The above definition is the expected one: each (generic) rule in Rls yields, for each substitution of the variables in the rule and for each choice of the actions 
fulfilling the side-condition, an inference of the instance of the rule's conclusion from the instances of the rule's hypotheses.

Bisimilarity. We write rel for (term $\times$ term) set, the type of relations between terms, ranged over by $\theta, \eta, \xi$. The (monotonic) retract operator Retr :: rel $\Rightarrow$ rel, named so because it maps each $\theta$ to a relation retracted (w.r.t. transitions) back from $\theta$, is defined by: $\operatorname{Retr} \theta=\left\{(P, Q) .\left(\forall a, P^{\prime} . P \stackrel{a}{\rightsquigarrow} P^{\prime} \longrightarrow\left(\exists Q^{\prime} .\left(P^{\prime}, Q^{\prime}\right) \in\right.\right.\right.$ $\left.\left.\left.\theta \wedge Q \stackrel{a}{\rightsquigarrow} Q^{\prime}\right)\right) \wedge\left(\forall a, Q^{\prime} . Q \stackrel{a}{\rightsquigarrow} Q^{\prime} \longrightarrow\left(\exists P^{\prime} .\left(P^{\prime}, Q^{\prime}\right) \in \theta \wedge P \stackrel{a}{\rightsquigarrow} P^{\prime}\right)\right)\right\}$. The bisimilarity relation, bis :: rel, is the greatest fixed point of Retr.

Notice that we defined bisimilarity for open terms (i.e., terms possibly containing variables), while often in the literature both transition and bisimilarity are defined for closed terms only (with step and Retr defined by the same conditions as above, but acting on closed terms and relations on closed terms, respectively). However, for the de Simone format of our rules (as well as for more general formats, e.g., well-founded pure tyft [15]), transition does not bring any variables (in the sense that, if $P \stackrel{a}{\rightsquigarrow} P^{\prime}$, then the free variables of $P$ are among those of $P^{\prime}$ ) implying that two closed terms are bisimilar according to our definition iff they are bisimilar according to the aforementioned "closed" version.

Because of the particular format of the rules, bis is a congruence on terms. This is in fact true for rule formats more expressive than the one considered here 6 1532. However, we shall need to exploit a stronger property specific to the de Simone format, namely: whenever $\theta$ is a congruence, it follows that $\theta \cap(\operatorname{Retr} \theta)$ is also a congruence. Let, for any relation $\theta, \operatorname{congCl} \theta$ be its congruence closure. From the above, we infer a powerful "up to" coinduction rule (that is, up to bisimilarity and up to arbitrary contexts), due to de Simone [11] and Sangiorgi [34, improving on traditional coinduction:

Theorem 1. For all $\theta::$ rel, if $\theta \subseteq \operatorname{Retr}(\operatorname{congCl}(\theta \cup$ bis $))$, then $\theta \subseteq$ bis.

\section{The Raw Coinductive Proof System}

We now present the core of our original theoretical contribution: defining an incrementally-coinductive proof system for bisimilarity and proving it sound. We define the raw deduction relation $\mathrm{F}:: \mathbf{r e l} \Rightarrow \mathbf{r e l} \Rightarrow$ bool (with infix notation) inductively by the clauses:

\begin{tabular}{|c|c|c|}
\hline$(\mathrm{Ax})$ & $\forall \theta^{\prime} \in \Theta . \theta \mathrm{F} \theta^{\prime}($ Split $)$ & $\theta^{\prime} \cup \theta \mathrm{F} \theta^{\prime \prime}($ Coind $)$ \\
\hline$\theta \mathrm{F} \theta^{\prime}\left[\theta^{\prime} \subseteq \operatorname{congCl}(\theta \cup\right.$ bis $\left.)\right]$ & $\frac{\mathrm{F} \cup \Theta}{\theta}[\Theta \neq \emptyset]$ & {$\left[\theta^{\prime}\left[\theta^{\prime} \subseteq \operatorname{Retr} \theta^{\prime \prime}\right]\right.$} \\
\hline
\end{tabular}

$\theta \mathrm{F} \theta^{\prime}$ is eventually intended to mean: " $\theta$ implies $\theta^{\prime}$ modulo bisimilarity and congruence closure". Here is the intuitive reading of the rules (thinking of them as being applied backwards for expanding or discharging goals). (Ax) allows to deduce $\theta^{\prime}$ from $\theta$ right away. (Split) allows for splitting the goal according to a chosen partition of its conclusion. (Coind) is the interesting rule, and is the actual engine of the proof system. To get an intuitive grasp of this rule, let us first assume that $\theta=\emptyset$ (i.e., that $\theta$ is empty). Then the goal is to show $\theta^{\prime}$ included in 
congCl bis, i.e., in bis. For this, it would suffice that $\theta^{\prime} \subseteq \operatorname{Retr}\left(\theta^{\prime}\right)$; alternatively, we may "defer" the goal by coming up with an "interpolant" $\theta^{\prime \prime}$ such that $\theta^{\prime} \subseteq$ $\operatorname{Retr}\left(\theta^{\prime \prime}\right)$ and $\theta^{\prime}$ implies $\theta^{\prime \prime}$ modulo bisimilarity and congruence. (As we shall see in the next section, working symbolically with open terms provides natural interpolant candidates.) In case $\theta \neq \emptyset, \theta$ should be thought of temporally as the collection of auxiliary facts gathered from previous coinductive expansions.

Note that, for the aforementioned intention of the proof system, (Coind) is not sound by itself: regarded as applied backwards to a goal, it moves the conclusion $\theta^{\prime}$ to the hypotheses, creating a circularity. In other words, of course it is not true that the conjunction of $\theta^{\prime \prime} \subseteq \operatorname{cong} \mathrm{Cl}\left(\theta^{\prime} \cup \theta \cup\right.$ bis $)$ and $\theta^{\prime} \subseteq$ Retr $\theta^{\prime \prime}$ implies $\theta^{\prime} \subseteq \operatorname{cong} \mathrm{Cl}(\theta \cup$ bis $)$ for all $\theta, \theta^{\prime}, \theta^{\prime \prime}$. Yet, the proof system as a whole is sound in the following sense:

Theorem 2. If $\emptyset \mathrm{F} \theta$, then $\theta \subseteq$ bis.

In the remainder of this section, we outline the proof of this theorem.

(I) In order to gain more control on the proof system, we objectify it in a standard fashion, by considering proofs (i.e., proof trees) explicitly, at the object level (as opposed to merely implicitly as they appear in the inductive definition of $\mathrm{F}$ ). For this, we pick a sufficiently large type index, ranged over by $i$, and define the type prf, of proof trees, ranged over by $P f$, with constructors mirroring the clauses in the definition of $\mathrm{F}$ :

\section{DATATYPE prf $=A \times$ rel rel $\mid$ Split (index $\Rightarrow$ prf) rel rel $\mid$ Coind prf rel rel}

We let $P f s$ range over index $\Rightarrow$ prf. The pair of relations that a proof tree $P f$ "proves", which is $\left(\theta, \theta^{\prime}\right)$ when $P f$ has the one of the forms Ax $\theta \theta^{\prime}$, Split Pfs $\theta \theta^{\prime}$, or Coind $\operatorname{Pf} \theta \theta^{\prime}$, is denoted by proves $\operatorname{Pf}$. The conclusion-hypothesis dependencies and the side-conditions of the clauses defining $F$ are captured by the predicate correct :: $\mathbf{p r f} \Rightarrow \mathbf{b o o l}$, defined recursively as expected:

- $\operatorname{correct}\left(\operatorname{Ax} \theta \theta^{\prime}\right)=\left(\theta^{\prime} \subseteq \operatorname{congCl}(\theta \cup\right.$ bis $\left.)\right)$;

- $\operatorname{correct}\left(\right.$ Split Pfs $\left.\theta \theta^{\prime}\right)=$

$\left((\forall i\right.$. $\operatorname{correct}(P f s i) \wedge \mathrm{fst}(\operatorname{proves}(P f s i))=\theta) \wedge \bigcup i$. snd $\left.(\operatorname{proves}(P f s i))=\theta^{\prime}\right)$;

- correct (Coind Pf $\left.\theta \theta^{\prime}\right)=$

(correct $P f \wedge$ fst $($ proves $P f)=\theta^{\prime} \cup \theta \wedge \theta^{\prime} \subseteq \operatorname{Retr}(\operatorname{snd}($ proves $\left.P f))\right)$.

It is immediate that $\theta \mathrm{F} \theta^{\prime}$ holds iff $\exists P f$. correct $(P f) \wedge \operatorname{proves}(P f)=\left(\theta, \theta^{\prime}\right)$.

(II) Thus, it suffices to show that $\theta \subseteq$ bis whenever there exists a correct proof tree $P f$ such that $\operatorname{proves}(P f)=\left(\theta, \theta^{\prime}\right)$. For showing the latter, we introduce a couple of auxiliary concepts. Given $P f$, a label in $P f$ is a pair $\left(\theta, \theta^{\prime}\right)$ "appearing" in $P f$ - formally, we define labels :: prf $\Rightarrow(\mathbf{r e l} \times \mathbf{r e l})$ set by:

- labels $\left(\operatorname{Ax} \theta \theta^{\prime}\right)=\left\{\left(\theta, \theta^{\prime}\right)\right\}$;

- labels (Split Pfs $\left.\theta \theta^{\prime}\right)=\left\{\left(\theta, \theta^{\prime}\right)\right\} \cup \bigcup i$. labels $(P f s i)$;

- labels (Coind Pf $\left.\theta \theta^{\prime}\right)=\left\{\left(\theta, \theta^{\prime}\right)\right\} \cup$ labels Pf.

We let Left $P f$ denote the union of the lefthand sides of all labels in $P f$, and Right $P f$ the union of the righthand sides of all labels in $P f$.

Lemma 1. If $P f$ is correct, then Right $P f \subseteq \operatorname{congCl}(($ Left $P f) \cup$ bis $)$.

Lemma 2. If $P f$ is correct and fst(proves $P f) \subseteq \operatorname{Retr}($ Right $P f)$, then Left $P f \subseteq$ $\operatorname{Retr}($ Right $P f)$. 
Lemma 1 follows by an easy induction on proof trees. By contrast, Lemma 2 requires some elaboration - before getting into that, let us show how the two lemmas imply our desired fact. Assume that $\operatorname{Pf}$ is correct and proves $\operatorname{Pf}=(\emptyset, \theta)$. Then the hypotheses of both lemmas are satisfied by $P f$, and therefore (since also Retr is monotonic) Left $P f \subseteq \operatorname{Retr}($ Right $P f) \subseteq \operatorname{Retr}(\operatorname{congCl}(($ Left $P f) \cup$ bis $))$, implying, by Theorem 1] Left $P f \subseteq$ bis. With Lemma 1, we obtain Right $P f \subseteq$ congCl(bis), which means (given that bis is a congruence) Right $P f \subseteq$ bis. And since $\theta \subseteq$ Right $P f$, we obtain $\theta \subseteq$ bis, as desired.

It remains to prove Lemma 2. This lemma states a property of proof trees that depends on a hypothesis concerning their roots (i.e., the pair $\left(\theta, \theta^{\prime}\right)$ that they "prove"). The task of finding a strengthening of that hypothesis so that a direct proof by structural induction goes through seems rather difficult, if not impossible. We instead take the roundabout route of identifying an invariant satisfied on backwards paths in the proof trees whose roots satisfy our hypothesis. First, we define the notion of a path (independently of proof trees): a list $\left[\left(\theta_{0}, \theta_{0}^{\prime}\right), \ldots,\left(\theta_{m-1}, \theta_{m-1}^{\prime}\right)\right]$ is called a path if the following is true for all $n<m-1$ : either $\theta_{n+1}=\theta_{n}$, or $\theta_{n+1} \subseteq \operatorname{Retr}\left(\theta_{n+1}^{\prime}\right) \cup \theta_{n}$. Then one can verify the following: -(a) Fix $\xi$ :: rel. If $\left[\left(\theta_{0}, \theta_{0}^{\prime}\right), \ldots,\left(\theta_{m-1}, \theta_{m-1}^{\prime}\right)\right]$ is a path, $\theta_{0} \subseteq \operatorname{Retr} \xi$ and $\forall n<m . \theta_{n}^{\prime} \subseteq \xi$, then $\forall n<m . \theta_{n} \subseteq \operatorname{Retr} \xi$. (By easy induction on $n$.)

-(b) If $P f$ is correct, $\operatorname{proves}(P f)=\left(\theta, \theta^{\prime}\right)$, and $\left(\eta, \eta^{\prime}\right)$ is a label in $P f$, then there exists a path $\left[\left(\theta_{0}, \theta_{0}^{\prime}\right), \ldots,\left(\theta_{m-1}, \theta_{m-1}^{\prime}\right)\right]$ consisting of labels in $P f$ (i.e., such that $\left(\theta_{n}, \theta_{n}^{\prime}\right)$ are labels in $P f$ for all $\left.n<m\right)$ and connecting $\left(\theta, \theta^{\prime}\right)$ with $\left(\eta, \eta^{\prime}\right)$ (i.e., such that $\left(\theta_{0}, \theta_{0}^{\prime}\right)=\left(\theta, \theta^{\prime}\right)$ and $\left.\left(\theta_{m-1}, \theta_{m-1}^{\prime}\right)=\left(\eta, \eta^{\prime}\right)\right)$. (By induction on Pf.)

With these preparations, we can prove Lemma 2] Assume proves $(P f)=\left(\theta, \theta^{\prime}\right)$ and $\theta \subseteq \operatorname{Retr}($ Right $P f)$. Fix a label $\left(\eta, \eta^{\prime}\right)$ in $P f$. According to (b), there exists a path connecting $\left(\theta, \theta^{\prime}\right)$ with $\left(\eta, \eta^{\prime}\right)$ and going through labels in $P f$ only. Then the hypotheses of (a) are satisfied by the aforementioned path and $\xi=$ Right $P f$, and therefore all the lefthand sides of the pairs in this path are included in $\operatorname{Retr}($ Right $P f)$. In particular, $\eta \subseteq \operatorname{Retr}($ Right $P f)$. Since the choice of the label $\left(\eta, \eta^{\prime}\right)$ was arbitrary, it follows that Left $P f \subseteq \operatorname{Retr}($ Right $P f)$, as desired.

Remarks. (1) The soundness of $\mathrm{F}$ was established not locally (rule-wise), as is customary in soundness results, but globally, by analyzing entire proof trees. What the potential backwards applications of the clause (Coind) do is to improve the candidate relation for the coinductive argument. In the end, as shown by the proof of Theorem 2, the (successful) relation is synthesized by putting together the righthand sides of all labels in the proof tree.

(2) The proof system represented by $F$ is not a typical syntactic system, but contains semantic intrusions - in effect, the system is complete already by its axiom $(\mathrm{Ax})$, which allows for an instantaneous "oracle proof" that the considered relation is included in bisimilarity. But of course, the realistic employment of this system will appeal to such instantaneous proofs only through the available (already proved) lemmas. (Thus, the purpose of including bis in the side-condition of $(\mathrm{Ax})$ was not to ensure completeness (in such a trivial manner), but to allow the usage of previously known facts about bisimilarity.) A more syntactic 
and syntax-driven system for terms (also featuring oracles though, for the same reason as this one) will be presented in the next section.

\section{Deduction of Universally Quantified Bisimilarity Equations}

Next we introduce a deduction system for term equalities, where, as before, we interpret equality as bisimilarity, but now we interpret the occurring variables as being universally quantified over the domain of terms.

Universal bisimilarity, ubis :: rel, is defined as follows: $\left(U, U^{\prime}\right) \in$ ubis iff $\left(U[\tau], U^{\prime}[\tau]\right) \in$ bis for all substitutions $\tau::$ var $\Rightarrow$ term. Thus, e.g., given distinct variables $X$ and $Y$ and an opsym $f,($ Op $f[][\operatorname{Var} X, \operatorname{Var} Y], O p f[][\operatorname{Var} Y, \operatorname{Var} X])$ $\in$ ubis is equivalent to $\forall U, V$ :: term. (Op $f[][U, V]$, Op $f[][V, U]) \in$ bis.

Matched derived rules. Derived rules appear by composing primitive rules (i.e., the de Simone rules in $R l s$ ) within maximal composition chains. I.e., they come from considering, in the SOS system, derivation trees that are completely backwards-saturated (in that their leaves involve only variables as sources and targets) and then forgetting the intermediate steps in these trees. A derived rule may not be amenable (hence not de Simone), but will always be sane. We shall let $d r l$ denote derived rules, keeping the symbol $r l$ for primitive rules.

We are interested in constructing all derived rules that are matched by a given term $U$ in such a way that $U$ becomes the source of the conclusion of the derived rule; in doing so, we also care about avoiding any overlap between the freshly generated variables (required to build the rules) and the variables of another given term $V$ (that we later wish to prove universally bisimilar with $U$ ). We thus introduce the operator $\mathrm{mdr}:: \operatorname{term} \Rightarrow \operatorname{term} \Rightarrow$ rule set, read "matched derived rules", such that, given $U, V$ : term, mdr $V U$ is the set of all the derived rules with $U$ as the source of their conclusion and with "the Ys" fresh for $V$. We write $\operatorname{mdr}_{V} U$ instead of $\operatorname{mdr} V U$.

The definition of $\mathrm{mdr}$ is both intuitive and standard (and was already sketched in the pioneering paper [1]), but its formalities are very technical, due to the need to avoid name overlapping and compose side-conditions. Here, we count on its understanding by examples and by its abstract properties, but App. A in 28 gives the general definition. (In 64, , where what we call "matched derived rules" are called "ruloids", mdr is not even defined, but rather the existence of such an operator satisfying suitable properties (essentially the same with what we call below soundness and completeness of the matched derived rules) is proved.)

Running example (continued). We again assume that all the variables $X, Y$ etc. that we refer to below are fixed distinct variables.

- $\operatorname{mdr}{ }_{! X}(X \mid ! X)$, the set of derived rules matched by $X \mid ! X$ and with "the Ys" avoiding the variables of $! X$, consists of $\left\{\mathrm{DRL}_{1}, \mathrm{DRL}_{2}, \mathrm{DRL}_{3}, \mathrm{DRL}_{4}\right\}$ (see below); - $\operatorname{mdr}_{X \mid ! X}(! X)$, the set of derived rules matched by $! X$ and with "the Ys" avoiding the variables of $X \mid ! X$, consists of $\left\{\mathrm{DRL}_{5}, \mathrm{DRL}_{6}\right\}$ (given below). 


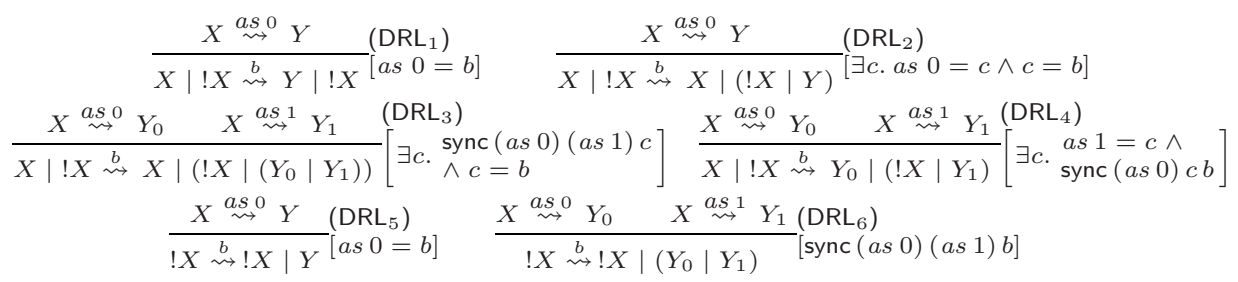

Remarks. (1) Because the term ! X has only depth 1, the matched derived rules $\mathrm{DRL}_{5}, \mathrm{DRL}_{6}$ are essentially the primitive rules REPL, REPLS. Moreover, DRL 1 was obtained by a single (backwards) application of the rule PARL.

(2) Each of $\mathrm{DRL}_{2}, \mathrm{DRL}_{3}, \mathrm{DRL}_{4}$ arises from the composition of two primitive rules. For example, $\mathrm{DRL}_{3}$ is obtained by applying PARR, and then applying REPLS to the resulted hypothesis:

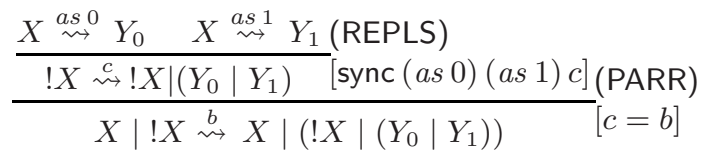

The side-condition of $\mathrm{DRL}_{3}$ is obtained by composing (essentially as relations) the two side-conditions, of PARR and REPLS, yielding existential quantification over $c$. Of course, the side-conditions of $\mathrm{DRL}_{2}, \mathrm{DRL}_{3}, \mathrm{DRL}_{4}$ can be readily simplified to the equivalent forms as $0=b$, sync (as 0$)(a s 1) b$ and again sync (as 0) (as 1) b, but eliminating the existential quantifiers may not be possible in general - recall that side-conditions are arbitrary predicates.

The only property we care about concerning elements $d r l$ of $\operatorname{mdr}_{V} U$ w.r.t. $V$ is that theYs $(d r l)$ are all distinct from the variables of $V$. On the other hand, concerning the relationship between $\operatorname{mdr}_{V} U$ and $U$, we have the crucial facts of soundness and completeness w.r.t. transition:

- For all $d r l \in \operatorname{mdr}_{V} U, d r l$ is sound, i.e.: for all $\tau$ :: var $\Rightarrow \operatorname{term}$, as :: nat $\Rightarrow$ act, and $b::$ act, if $\tau(($ theXXs $d r l) ! j) \stackrel{\text { as } j}{\sim} \tau(($ theYs $d r l) ! j)$ for all $j<$ length(theYs $d r l$ ) and side $d r l$ as $b$ holds, then (theS $d r l)[\tau] \stackrel{b}{\rightsquigarrow}$ (theT $d r l)[\tau]$.

- $\operatorname{mdr}_{V} U$ is complete for inference of transitions with sources that match $U$, i.e.: for all $\tau:: \operatorname{var} \Rightarrow \operatorname{term}, b::$ act and $Q::$ term such that $U[\tau] \stackrel{b}{\rightsquigarrow} Q$, there exist $d r l \in \operatorname{mdr}_{V} U, \tau^{\prime}:: \operatorname{var} \Rightarrow$ term and as :: nat $\Rightarrow$ act such that:

- $\tau^{\prime}$ coincides with $\tau$ on vars $U$ (hence $U[\tau]=U\left[\tau^{\prime}\right]$ );

- $\tau^{\prime}(($ theXXs $d r l) ! j) \stackrel{\text { as } j}{\sim} \tau^{\prime}(($ theYs $d r l) ! j)$ for all $j<$ length(theYs $\left.d r l\right)$;

- side drl as $b$ holds;

- (theT $d r l)\left[\tau^{\prime}\right]=Q$ (and also, remember that theS $\left.d r l=U\right)$.

Deduction of universal bisimulation. An equation will be simply a pair of terms, written $U \cong V$, and we write equation for the type of equations. (Note that rel is the same as equation set.) Our goals will consist of pairs (set of equations) - equation, where all equations shall be thought of as being universally quantified. We shall mostly use $S, T, U, V$ for terms thought of as patterns, and $P, Q, R$ for terms thought of as instances. 
Given $U, U^{\prime}:: \operatorname{term}, G:: \operatorname{mdr}_{U^{\prime}} U \Rightarrow \operatorname{mdr}_{U} U^{\prime}$, and $g:: \prod_{d r l \in \operatorname{mdr}_{U^{\prime}} U}\{0, \ldots$, length(theXXs $(G d r l))-1\} \Rightarrow\{0, \ldots$, length(theXXs $d r l)-1\}$, we define the predicate simul $U U^{\prime} G g$, read " $U$ is (one-step-) simulated by $U^{\prime}$ via $G$ and $g$ ", to mean that, for all $d r l \in \operatorname{mdr}_{U} U^{\prime}$, the following holds: Assume $d r l$ has the form

$$
\left.\frac{X X_{0} \stackrel{\text { as } 0}{\rightsquigarrow} Y_{0}, \ldots, X X_{n-1} \stackrel{\text { as }(n-1)}{\rightsquigarrow} Y_{n-1}}{S \stackrel{b}{\rightsquigarrow} T} \text { [side drl as } b\right]
$$

and $d r l^{\prime}=G d r l$ has the form

$$
\frac{X X_{0}^{\prime} \stackrel{\text { as } 0}{\rightsquigarrow} Y_{0}^{\prime}, \ldots, X X_{n^{\prime}-1}^{\prime} \stackrel{\text { as }}{\left.n^{\prime}-1\right)} Y_{n^{\prime}-1}^{\prime}}{S^{\prime} \stackrel{b}{\rightsquigarrow} T^{\prime}}\left[\text { side } d r l^{\prime} \text { as } b\right]
$$

(and therefore $g d r l::\left\{0, \ldots, n^{\prime}-1\right\} \Rightarrow\{0, \ldots, n-1\}$ ) Then:

- (1) $X X_{g d r l j}=X X_{j}^{\prime}$ (i.e., syntactically equal, as variables) for all $j<n^{\prime}$.

- (2) $\forall a s::$ nat $\Rightarrow$ act, $b::$ act. side $d r l$ as $b \longrightarrow$ side $(G d r l)(a s \circ(g d r l)) b$.

Given the rules $d r l$, of the form $(*)$, and $d r l^{\prime}$, of the form $(* *)$, and given $h::\left\{0, \ldots, n^{\prime}-1\right\} \Rightarrow\{0, \ldots, n-1\}$, we define newGoal $d r l d r l^{\prime} h$ to be the equation $T \cong T^{\prime}\left[\left(Y_{j}^{\prime} / Y_{h j}\right)_{j<n^{\prime}}\right]$, where $\left(Y_{j}^{\prime} / Y_{h j}\right)_{j<n^{\prime}}$ is a substitution that maps each variable $Y_{j}^{\prime}$ to the variable $Y_{h j}$ (more accurately, to the term $\operatorname{Var} Y_{h j}$ ).

simul and newGoal will work in tandem in our deduction system as follows: Given a goal $U \cong U^{\prime}$, we wish to prove $U$ and $U^{\prime}$ universally bisimilar. For this, we should show that, for any continuation of an instance of $U$, there exists a bisimilar continuation of an instance of $U^{\prime}$ (and vice versa, but next we ignore the "vice versa" part). By the completeness of mdr, any transition of an instance of $U$ is given by a derived rule $d r l$ in $\operatorname{mdr}_{U^{\prime}} U$. By the soundness of $\mathrm{mdr}$, for finding a transition of an instance of $U^{\prime}$ that simulates that of $U$, it would suffice to find for $d r l$ a derived rule in $d r l^{\prime}$ which is possible whenever $d r l$ is possible. Thus, we first need a map $G:: \operatorname{mdr}_{U^{\prime}} U \Rightarrow \operatorname{mdr}_{U} U^{\prime}$ (giving the $d r l^{\prime}$ for each $\left.d r l \in \operatorname{mdr}_{U^{\prime}} U\right)$, and then, for each $d r l$, a justification of the possibility of $G d r l$ in terms of that of $d r l$. Now, possibility of (a transition along) a derived rule is given by its (formal) hypotheses and its side conditions. Hence, a justification of the possibility of $G d r l$ in terms of the possibility of $d r l$ can be given by a map from the hypotheses of $G d r l$ to those of $d r l$ that preserves the sources (which are variables) and yields an implication between the side conditions - this is formally achieved by a function $g:: \prod_{d r l \in \operatorname{mdr}_{U^{\prime}} U}\{0, \ldots$, length(theXXs $\left.(G d r l))-1\right\} \Rightarrow$ $\{0, \ldots$, length(theXXs $d r l)-1\}$ that, together with $G$, satisfies the conditions defining simul $U U^{\prime} G g$. Moreover, we have to prove that, for each combination $(d r l, G d r l)$, the resulted continuations of the presumptive instances of $U$ and $U^{\prime}$ are again bisimilar - we obtain a newGoal $d r l(G d r l)(g d r l)$ for each such combination (note that generating this new goal has to take into consideration the dispatching of formal hypotheses performed by $g d r l$, meaning that we also have to substitute some "Ys"). Finally, the incremental nature of our coinduction (inherited from the previous section) shows up: for proving each of the new goals, we may assume the old goal, $U \cong U^{\prime}$.

We are led to the deduction relation $\vdash::$ equation $\underline{\text { set }} \Rightarrow$ equation $\Rightarrow$ bool (with infix notation), defined inductively by the following clauses: 


$$
\begin{aligned}
& \text { (Eqnl) } \\
& \overline{\theta \vdash U \cong U^{\prime}}\left[\theta \cup \text { bis } \vdash_{\text {eq }} U \cong U^{\prime}\right] \\
& \forall d r l \in \operatorname{mdr}_{U^{\prime}} U . \theta \cup\left\{U \cong U^{\prime}\right\} \vdash \text { newGoal } d r l(G d r l)(g d r l) \\
& \frac{\forall d r l^{\prime} \in \operatorname{mdr}_{U} U^{\prime} \cdot \theta \cup\left\{U \cong U^{\prime}\right\} \vdash \text { newGoal } d r l^{\prime}\left(G^{\prime} d r l^{\prime}\right)\left(g^{\prime} d r l^{\prime}\right)}{\theta \vdash U \cong U^{\prime}}\left[\begin{array}{l}
\text { (Coind) } \\
\text { simul } U U^{\prime} G g \\
\text { simul } U^{\prime} U G^{\prime} g^{\prime}
\end{array}\right]
\end{aligned}
$$

In the side-condition at $($ Eqnl $), \vdash_{\text {eq }}$ is standard equational-logic deduction. We include bis among the hypotheses, because we wish to allow any known facts about bisimilarity to "help" $\vdash$-deduction, including facts obtained by means other than $\vdash$. Again, due to circularity (moving goals to the hypotheses), a rule like (Coind) cannot be sound in itself, but again we have global soundness:

Theorem 3. If $\emptyset \vdash U \cong U^{\prime}$, then $\left(U, U^{\prime}\right) \in$ ubis.

Proof sketch. We use the soundness of $\mathrm{F}$ (Theorem 1) together with the rules defining $\vdash$ being simulated by those defining $F$. Namely, we show, by induction on $\vdash$, that $\theta \vdash U \cong U^{\prime}$ implies sstvsmCl$(\theta) \vdash \operatorname{sstvsmCl}\left(\left\{\left(U, U^{\prime}\right)\right\}\right)$, where sstvsmCl :: rel $\Rightarrow$ rel gives the substitutive and symmetric closure of a relation, i.e., sstvsmCl $(\xi)=\{(S[\sigma], T[\sigma]) . \sigma:: \operatorname{var} \Rightarrow \operatorname{term},(S, T) \in \xi \vee(T, S) \in \xi\}$.

If $\theta \vdash U \cong U^{\prime}$ followed by an application of (Eqnl), then $\operatorname{sstvsmCl}(\theta) \mathrm{F}$ sstvsmCl$\left(\left\{\left(U, U^{\prime}\right)\right\}\right)$ follows applying the F-clause $(\mathrm{Ax})$, since the equational closure coincides with the substitutive symmetric closure of the congruence closure.

Assume now $\theta \vdash U \cong U^{\prime}$ followed by (Coind), meaning that there exist $G, g, G^{\prime}, g^{\prime}$ such that: (i) simul $U U^{\prime} G g$; (ii) $\forall d r l \in \operatorname{mdr}_{U^{\prime}} U . \theta \cup\left\{U \cong U^{\prime}\right\} \vdash$ newGoal $d r l$ ( $G d r l)(g d r l)$; (iii) simul $U^{\prime} U G^{\prime} g^{\prime}$; (iv) $\forall d r l^{\prime} \in \operatorname{mdr}_{U} U^{\prime} . \theta \cup$ $\left\{U \cong U^{\prime}\right\} \vdash$ newGoal $d r l^{\prime}\left(G^{\prime} d r l^{\prime}\right)\left(g^{\prime} d r l^{\prime}\right)$. Then, by the induction hypothesis: - $\forall d r l \in \operatorname{mdr}_{U^{\prime}} U$. sstvsmCl$\left(\theta \cup\left\{U \cong U^{\prime}\right\}\right) \mathrm{F} \operatorname{sstvsmCl}(\{$ newGoal $d r l(G d r l)$ $(g d r l)\})$.

- $\forall d r l^{\prime} \in \operatorname{mdr}_{U} U^{\prime} . \operatorname{sstvsmCl}\left(\theta \cup\left\{U \cong U^{\prime}\right\}\right) \mathrm{F}$ sstvsmCl(newGoal $d r l^{\prime}\left(G^{\prime} d r l^{\prime}\right)$ $\left.\left(g^{\prime} d r l^{\prime}\right)\right)$.

Let $\theta^{\prime}=\operatorname{sstvsmCl}\left(\left\{\left(U, U^{\prime}\right)\right\}\right)$ and let $\theta^{\prime \prime}=\{$ newGoal $d r l(G d r l)(g d r l) . d r l \in$ $\left.\operatorname{mdr}_{U^{\prime}} U\right\} \cup\left\{\right.$ newGoal $\left.d r l^{\prime}\left(G^{\prime} d r l^{\prime}\right)\left(g^{\prime} d r l^{\prime}\right) . d r l^{\prime} \in \operatorname{mdr}_{U} U^{\prime}\right\}$. The crucial thing to notice is that, since simul $U U^{\prime} G g$ and simul $U^{\prime} U G^{\prime} g^{\prime}$ hold, sstvsmCl$\left(\left\{\left(U, U^{\prime}\right)\right\}\right)$ $\subseteq \operatorname{Retr} \theta^{\prime}$ also holds - and the paragraph right before introducing $\vdash$ can be regarded as an informal justification for why this is true. Therefore, $\theta^{\prime \prime}$ is an "interpolant" for applying the F-clause (Coind). Indeed, applying the F-clause (Split) to (1) and (2), we obtain $\theta^{\prime} \cup \theta \vdash \theta^{\prime \prime}$ and then, by the $\mathrm{F}$-clause (Coind), we obtain $\theta \mathrm{F} \theta^{\prime}$, as desired.

Running example (finished). We are now ready to make rigorous the proof of $\forall P$ :: term. $(P \mid ! P, ! P) \in$ bis presented in the introduction. Consider the following four proof trees of depth 0 (later referred to as $P f_{1}, P f_{2}, P f_{3}, P f_{4}$ ) where we list the side-conditions for (Eqnl) as hypotheses: 


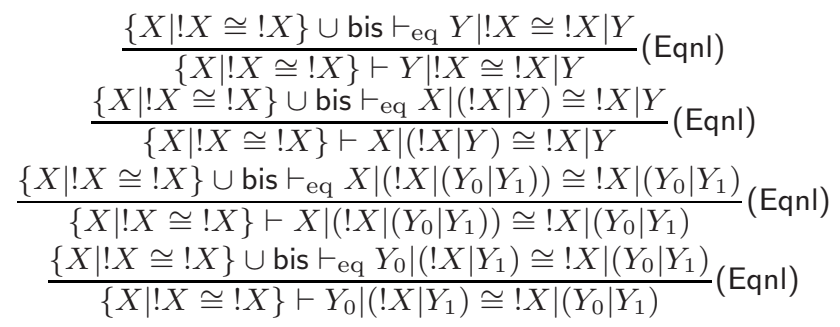

Then our final proof (tree) is:

$$
\frac{P f_{1} \quad P f_{2} \quad P f_{3} \quad P f_{4}}{\emptyset \vdash X \mid ! X \cong ! X} \text { (Coind) }
$$

Explanations. At (Coind), we took:

- $G$ to map $\mathrm{DRL}_{1}$ and $\mathrm{DRL}_{2}$ to $\mathrm{DRL}_{5}$, and to map $\mathrm{DRL}_{3}$ and $\mathrm{DRL}_{4}$ to $\mathrm{DRL}_{6}$;

- $g \mathrm{DRL}_{1}$ and $g \mathrm{DRL}_{2}$ to be the identity on $\{0\}$, and $g \mathrm{DRL}_{3}$ and $g \mathrm{DRL}_{4}$ to be the identity on $\{0,1\}$;

- $G^{\prime}$ to map $\mathrm{DRL}_{5}$ to $\mathrm{DRL}_{1}$, and to map $\mathrm{DRL}_{6}$ to $\mathrm{DRL}_{3}$;

- $g^{\prime} \mathrm{DRL}_{5}$ to be the identity on $\{0\}$, and $g^{\prime} \mathrm{DRL}_{6}$ to be the identity on $\{0,1\}$.

(Note that any function $G^{\prime}$ mapping $\mathrm{DRL}_{5}$ to either $\mathrm{DRL}_{1}$ or $\mathrm{DRL}_{2}$ and $\mathrm{DRL}_{6}$ to either $\mathrm{DRL}_{3}$ or $\mathrm{DRL}_{4}$ together with $g^{\prime}$ as above would lead to a valid proof.)

Here is why we end up with the above four proof tasks after applying (Coind):

newGoal DRL ${ }_{1}\left(G \mathrm{DRL}_{1}\right)\left(g \mathrm{DRL}_{1}\right)=$ newGoal DRL $\mathrm{DRL}_{5}(\lambda i . i)=Y|! X \cong ! X| Y$

newGoal DRL ${ }_{2}\left(G \mathrm{DRL}_{2}\right)\left(g \mathrm{DRL}_{2}\right)=$ newGoal DRL $\mathrm{DRL}_{5}(\lambda i . i)=X|(! X \mid Y) \cong ! X| Y$;

newGoal DRL ${ }_{3}\left(G \mathrm{DRL}_{3}\right)\left(g \mathrm{DRL}_{3}\right)=$ newGoal DRL $\mathrm{DRL}_{6}(\lambda i . i)=X\left|\left(! X \mid\left(Y_{0} \mid Y_{1}\right)\right) \cong ! X\right|\left(Y_{0} \mid Y_{1}\right)$;

newGoal DRL ${ }_{4}\left(G \mathrm{DRL}_{4}\right)\left(g \mathrm{DRL}_{4}\right)=$ newGoal DRL $\mathrm{DRL}_{6}(\lambda i . i)=Y_{0}\left|\left(! X \mid Y_{1}\right) \cong ! X\right|\left(Y_{0} \mid Y_{1}\right)$;

newGoal DRL ${ }_{5}\left(G^{\prime} \mathrm{DRL}_{5}\right)\left(g^{\prime} \mathrm{DRL}_{5}\right)=$ newGoal DRL $\mathrm{DRL}_{1}(\lambda i . i)=Y|! X \cong ! X| Y$;

newGoal DRL ${ }_{6}\left(G^{\prime} \mathrm{DRL}_{6}\right)\left(g^{\prime} \mathrm{DRL}_{6}\right)=$ newGoal DRL ${ }_{6} \operatorname{DRL}_{3}(\lambda i . i)=X\left|\left(! X \mid\left(Y_{0} \mid Y_{1}\right)\right) \cong ! X\right|\left(Y_{0} \mid Y_{1}\right)$.

The side-conditions of (Coind) are immediately checkable. E.g., for simul $(X \mid ! X)(! X) G g$, we need to check the following trivial facts:

- w.r.t. condition (1) (in the definition of simul): that $X=X$.

- w.r.t. condition (2): that each of the following are pairwise equivalent:

- as $0=b$ and as $0=b$;

- $\exists c$. as $0=c \wedge c=b$ and as $0=b$;

- $\exists c$. sync (as 0$)$ (as 1$) c \wedge c=b$ and sync (as 0$)$ (as 1$) b$;

- $\exists$ c. as $1=c \wedge \operatorname{sync}($ as 0$) c b$ and sync (as 0$)$ (as 1 ) $b$.

At (Eqnl) in all the four immediate subtrees of the main proof tree, we considered the fact (assumed previously proved) that $\{X|Y \cong Y| X,(X \mid Y) \mid Z \cong$ $X \mid(Y \mid Z)\} \subseteq$ bis, hence what we really used was equational-logic deduction from $\{X|! X \cong ! X, X| Y \cong Y|X,(X \mid Y)| Z \cong X \mid(Y \mid Z)\}$, which easily discharges the equational side-conditions of the axioms, finalizing the proof.

The above proof does not display any non-trivial "dispatch" function $g$ in the (Coind) rule application. In general however, it is not guaranteed that the formal hypotheses of two obtained derived rules (from the two terms of the goal) that one wishes to pair come in the same order, nor that these rules have the 
same number of hypotheses. (See the proof of commutativity of "" from App. $\mathrm{B}$ in [28].)

\section{Concluding Remarks}

We have developed and formalized in Isabelle a proof system for process algebra where bisimilarity is proved incrementally, while exploring and expanding the goal, without requiring an a priori constructed bisimulation relation. Our results apply to a wide class of process algebras.

Related work. Unique fixpoint induction for CCS and its variants [23 17/25] is an early notion of proof-theoretic circularity for coinduction applicable to situations where circularity is explicit in the SOS by means of (guarded) fixpoint equations. We conjecture that unique fixpoint induction in an instance of our incremental coinduction.

We had two major sources of inspiration. First, the idea of circular coinduction (CC for short) in the context of algebraic specifications. It was introduced in [14] in the behavioral specification language BOBJ [1, and then also implemented axiomatically in (generic) Isabelle under the "supervision" of the CoCASL specification language [16] and in Maude 9] as the circular coiductive prover CIRC 201931 . A comparison of our proof system with CC is somewhat difficult to sketch, as it has to deal with different technical settings and to balance the advantages of both generality and specialization. To simplify the discussion, we shall implicitly assume a back-and-forth translation between SOS specifications and the coalgebraic and behavioral specifications required by the $\mathrm{CC}$ settings. Our proof system is in a sense more general and in a sense more specialized.

It is more general in that it applies to nondeterministic processes, not handled by $\mathrm{CC}$ (e.g., the running example in this paper is not approachable in $\mathrm{CC}$, not even interactively). On the other hand, CIRC, based on rewriting logic, could employ the results presented here in order to extend $\mathrm{CC}$ with nondeterminism 2 Also, CoCASL as a specification language has the expressive power required to deal with process algebra and nondeterminism, hence to support a version of $\mathrm{CC}$ for nondeterministic systems. However, it is the determinism of CC that allows for partial automation, admirably illustrated by CIRC. (Our formalized system, once fine-tuned into a tool, will also allow automation for deterministic, and, to some extent, finitely-branching cases - see below the discussion on future work.)

It is more specialized in that the deterministic instances of our setting are more restricted than what $\mathrm{CC}$ can handle (in particular, e.g., deterministic lookahead, not approachable here, is unproblematic in CC). On the other hand, our powerful coinduction "up to", underneath arbitrary contexts (not supported by CC) is possible precisely because of this restriction.

\footnotetext{
${ }^{2}$ Which is not to say our proof system is a minor variation of CC - nondeterminism (technically, the interplay between our rules (Split) and (Coind) from Sec. 3) represented the main difficulty in our soundness proof.
} 
Finally, our coinductive technique is presented in a logical form, as a proof system, like in [31, and not as an algorithm like in the other cited works on CC. In [31, logical form is achieved through the introduction of so-called freezing operators, hard to justify logically - with this respect, our proof system has the advantage of "purity" 3 (Here we should also remark some less related work: circular systems in logical form were also developed in [710] for first-order logic and the $\mu$-calculus, respectively.)

The second major source of inspiration was the notion of coinduction proofs up to bisimilarity and arbitrary contexts, introduced in 1124 and developed in 34.35. This idea also appears in a general coalgebraic setting in [5] and is illustrated by extensive examples in, e.g., 33. The convenience of performing unrestricted equational reasoning relies essentially on the "up to" coinduction principle, Theorem 1 .

Other related work includes frameworks for bisimilarity of open terms in [2984 (also building on the seminal work from [1]), where open terms are considered universally quantified, as we do in this paper for universal bisimilarity. Our soundness result for $\vdash$ w.r.t. universal bisimilarity, Theorem 3 , could have been more sharply phrased: on one hand, as a soundness result w.r.t. the notion of bisimulation under formal hypotheses from 1129; on the other, w.r.t. to the relation from 4] (which is essentially universal bisimilarity in any conservative extension of the SOS system). All works cited in this paragraph discuss non-incremental proof systems, where the desired bisimulation relation needs to be fed by the user.

Descriptions of more or less automatic software tools for proving bisimilarity in process algebra abound in the literature - see 18 21] for overviews. While most of these tools are dedicated to (and optimized for) particular process algebras (and many to finite-state systems), ECRINS [12] is based precisely on generic process algebra in de Simone format, meaning that the results of this paper on incremental coinduction apply directly to that setting (and, interestingly, a form of coinduction that "attempts to add more couples to the [previously specified] relation" is indicated in [12] as a direction for future research, to our knowledge not pursued so far). Finally, in Coq [2], the interaction between its generalpurpose support for building proofs and its coinductive types (as illustrated, e.g., in [13]) also leads to a form of incremental coinduction whose relationship with our approach is yet to be understood.

Future work. The de Simone SOS format is already fairly general, covering a wide range of process algebras, including CCS-like process algebras with guarded recursion, and even de Simone systems under weak bisimilarity (since for them weak bisimilarity can be regarded as strong bisimilarity for trace-based de Simone systems, as suggested in [27]). An extension of our incremental coinductive technique to more general formats such as GSOS [6] or tyft/tyxt [15] is of course

\footnotetext{
${ }^{3}$ In a sense, what these freezing operators do is to guard against coinduction up-to, not sound in general. So again, our logical system achieves convenience because of specialization, i.e., by sacrificing some generality.
} 
desirable. Another direction for generalization is the allowance of bindings in the syntax of terms, including $\pi$-calculus-like bindings featuring scope extrusion (thus generalizing HOL-based settings for $\pi$-calculus such as [2230]).

In our proof system for universal bisimilarity, $\vdash$, one has to come up with suitable dispatch functions $G, g, G^{\prime}, g^{\prime}$ at each application of the coinduction rule (Coind), and therefore (Coind) is not syntax-directed per se, hence not trivially automatable - this is inherent in the hardness of bisimilarity in our general setting. However, (Coind) does allow to decompose the goal symbolically, without asking the user to decide for a global bisimilarity candidate instead, assisted by the powerful Isabelle classical reasoner and simplifier (able to discharge the typically simple goals resulted from chaining side-conditions), the user can explore various choices of the dispatch functions by analyzing the derived rules. Moreover, for systems with finite number of rules one can write an Isabelle tactic that tries all the combinations of dispatch functions. While this would require exponential time, it may still be feasible for cases of interest, since the time-complexity is a function of the symbolic branching of process patterns (determined by logical formulas obtained from side-conditions), and not of the actual branching of processes given by all possible instances of the rules. For the general case, we should aim at organizing our formalization (by means of proof tactics and pretty-printers) into a partly-interactive partly-automatic tool. The advantages of such a tool will of course include the generality of its scope and the fact that, unlike most other tools, it would be (a priori) formally certified.

Acknowledgments. We thank the reviewers for the careful and insightful reading of this paper and for pointing out related work. The first author is grateful to Grigore Roşu for many hours of discussion on his circular coinduction technique.

\section{References}

1. BOBJ, http://cseweb.ucsd.edu/groups/tatami/bobj

2. The Coq proof assistant, http://coq.inria.fr

3. Isabelle, http://www.cl.cam.ac.uk/research/hvg/Isabelle

4. Aceto, L., Cimini, M., Ingolfsdottir, A.: A bisimulation-based method for proving the validity of equations in GSOS languages. To appear in Electr. Proc. Theor. Comput. Sci.

5. Bartels, F.: Generalised coinduction. Math. Struct. Comp. Sci. 13(2), 321-348 (2003)

6. Bloom, B., Istrail, S., Meyer, A.R.: Bisimulation can't be traced. J. ACM 42(1), 232-268 (1995)

7. Brotherston, J.: Cyclic proofs for first-order logic with inductive definitions. In: Beckert, B. (ed.) TABLEAUX 2005. LNCS (LNAI), vol. 3702, pp. 78-92. Springer, Heidelberg (2005)

8. Bruni, R., de Frutos-Escrig, D., Martí-Oliet, N., Montanari, U.: Bisimilarity congruences for open terms and term graphs via Tile Logic. In: Palamidessi, C. (ed.) CONCUR 2000. LNCS, vol. 1877, pp. 259-274. Springer, Heidelberg (2000) 
9. Clavel, M., Durán, F.J., Eker, S., Lincoln, P., Martí-Oliet, N., Meseguer, J., Quesada, J.F.: The Maude system. In: Narendran, P., Rusinowitch, M. (eds.) RTA 1999. LNCS, vol. 1631, pp. 240-243. Springer, Heidelberg (1999)

10. Dam, M., Gurov, D.: $\mu$-calculus with explicit points and approximations. J. Log. Comput. 12(2), 255-269 (2002)

11. de Simone, R.: Higher-level synchronizing devices in MEIJE-SCCS. Theor. Comput. Sci. 37, 245-267 (1985)

12. Doumenc, G., Madelaine, E., de Simone, R.: Proving process calculi translations in ECRINS: The pureLOTOS $\rightarrow$ MEIJE example. Technical Report RR1192, INRIA (1990), http://hal .archives-ouvertes.fr/inria-00075367/en/

13. Giménez, E.: An application of co-inductive types in Coq: Verification of the alternating bit protocol. In: Berardi, S., Coppo, M. (eds.) TYPES 1995. LNCS, vol. 1158, pp. 135-152. Springer, Heidelberg (1996)

14. Goguen, J.A., Lin, K., Roşu, G.: Circular coinductive rewriting. In: ASE 2000, pp. 123-132 (2000)

15. Groote, J.F., Vaandrager, F.: Structured operational semantics and bisimulation as a congruence. Inf. Comput. 100(2), 202-260 (1992)

16. Hausmann, D., Mossakowski, T., Schröder, L.: Iterative circular coinduction for coCASL in Isabelle/HOL. In: Cerioli, M. (ed.) FASE 2005. LNCS, vol. 3442, pp. 341-356. Springer, Heidelberg (2005)

17. Hennessy, M., Lin, H.: Proof systems for message-passing process algebras. Formal Asp. Comput. 8(4), 379-407 (1996)

18. Inverardi, P., Priami, C.: Automatic verification of distributed systems: The process algebra approach. Formal Methods in System Design 8(1), 7-38 (1996)

19. Lucanu, D., Goriac, E.-I., Caltais, G., Roşu, G.: CIRC: A behavioral verification tool based on circular coinduction. In: Kurz, A., Lenisa, M., Tarlecki, A. (eds.) CALCO 2009. LNCS, vol. 5728, pp. 433-442. Springer, Heidelberg (2009)

20. Lucanu, D., Roşu, G.: CIRC: A circular coinductive prover. In: Mossakowski, T., Montanari, U., Haveraaen, M. (eds.) CALCO 2007. LNCS, vol. 4624, pp. 372-378. Springer, Heidelberg (2007)

21. Madelaine, E.: Verification tools from the CONCUR project, http://www-sop.inria.fr/meije/papers/concur-tools

22. Melham, T.F.: A mechanized theory of the pi-calculus in HOL. Nord. J. Comput. 1(1), 50-76 (1994)

23. Milner, R.: A complete inference system for a class of regular behaviours. J. Comput. Syst. Sci. 28(3), 439-466 (1984)

24. Milner, R.: Communication and concurrency. Prentice-Hall, Englewood Cliffs (1998)

25. Monroy, R., Bundy, A., Green, I.: On process equivalence = equation solving in ccs. J. Autom. Reasoning 43(1), 53-80 (2009)

26. Mousavi, M.R., Reniers, M.A., Groote, J.F.: SOS formats and meta-theory: 20 years after. Theor. Comput. Sci. 373(3), 238-272 (2007)

27. Popescu, A.: Weak bisimilarity coalgebraically. In: Kurz, A., Lenisa, M., Tarlecki, A. (eds.) CALCO 2009. LNCS, vol. 5728, pp. 157-172. Springer, Heidelberg (2009)

28. Popescu, A., Gunter, E.L.: Incremental pattern-based coinduction for process algebra and its Isabelle formalization. Technical Report, University of Illinois, http://hdl.handle.net/2142/14858

29. Rensink, A.: Bisimilarity of open terms. Inf. Comput. 156(1-2), 345-385 (2000) 
30. Röckl, C., Hirschkoff, D.: A fully adequate shallow embedding of the $\pi$-calculus in Isabelle/HOL with mechanized syntax analysis. J. Funct. Program. 13(2) (2003)

31. Roşu, G., Lucanu, D.: Circular coinduction: A proof theoretical foundation. In: Kurz, A., Lenisa, M., Tarlecki, A. (eds.) CALCO 2009. LNCS, vol. 5728, pp. 127 144. Springer, Heidelberg (2009)

32. Rutten, J.J.M.M.: Processes as terms: Non-well-founded models for bisimulation. Math. Struct. Comp. Sci. 2(3), 257-275 (1992)

33. Rutten, J.J.M.M.: Elements of stream calculus (an extensive exercise in coinduction). Electr. Notes Theor. Comput. Sci., 45 (2001)

34. Sangiorgi, D.: On the bisimulation proof method. Math. Struct. Comp. Sci. 8(5), 447-479 (1998)

35. Sangiorgi, D., Walker, D.: The $\pi$-calculus. A theory of mobile processes, Cambridge (2001) 